\title{
Cronobacter sakazakii DNA Detection in Cerebrospinal Fluid of a Patient with Amyotrophic Lateral Sclerosis Mimic Syndrome
}

\author{
Marianna Piombo ${ }^{a}$ Daniela Chiarello ${ }^{a}$ Marzia Corbetto ${ }^{a}$ \\ Giovanni DiPino ${ }^{a}$ d Giordano Dicuonzo ${ }^{b}$ Silvia Angeletti ${ }^{b}$ \\ Elisabetta Riva $^{c}$ Lucia DeFlorio $^{b}$ Fioravante Capone $^{\text {a, } d}$ \\ Vincenzo DiLazzaro ${ }^{\text {a, }}$ \\ ${ }^{a}$ Unit of Neurology, Neurophysiology, Neurobiology, Department of Medicine, University \\ Campus Bio-Medico of Rome, ${ }^{b}$ Clinical Pathology and Microbiology Laboratory and \\ 'Virology, Clinical Pathology and Microbiology Laboratory, University Hospital Campus \\ Bio-Medico of Rome, and ${ }^{d}$ Fondazione Alberto Sordi, Institute for Ageing, Rome, Italy
}

\section{Key Words}

Cronobacter sakazakii · Amyotrophic lateral sclerosis · Cerebrospinal fluid

\begin{abstract}
A 45-year-old male noticed progressive weakness of the right lower limb with gait disturbance. Over the following months, motor deficits worsened, spreading to the right upper limb. Electromyography showed active denervation in the upper and lower limb muscles. A diagnosis of amyotrophic lateral sclerosis (ALS) was made. About 2 years after symptom onset, gradual improvement occurred. Cerebrospinal fluid analysis performed about 3 years after the beginning of symptoms identified Cronobacter sakazakii. Since no other possible causes were identified, we suggest that an almost completely reversible ALS-like syndrome had been triggered by Cronobacter infection in our immunocompetent patient.
\end{abstract}

(C) 2015 The Author(s)

Published by S. Karger AG, Basel 


\section{Case Reports in Neurology}

Piombo et al.: Cronobacter sakazakii DNA Detection in Cerebrospinal Fluid of a Patient with Amyotrophic Lateral Sclerosis Mimic Syndrome

\section{Introduction}

Several cases of amyotrophic lateral sclerosis (ALS)-like syndrome have been described as an unusual manifestation of an infection of a few agents targeting motor neurons. An association between Cronobacter sakazakii infection and ALS-like syndrome has never been reported.

\section{Case Presentation}

In 2010, a 45-year-old male with an unremarkable medical history, noticed a progressive weakness of the right lower limb with gait disturbance. Over the following months, motor deficits worsened, spreading also to the right upper limb and impairing hand dexterous movements. In December 2010, he was admitted to the Neurological Department of a large university hospital. Spinal cord non-contrast-enhanced magnetic resonance imaging (MRI) showed multiple cervical spondylotic changes that were more pronounced at the C3-C4 level. Brain MRI, blood tests, and nerve conduction studies were normal. Electromyography (EMG) showed fibrillation and fasciculation potentials, positive sharp waves, enlarged motor units of increased duration, and a reduced interference pattern in the upper and lower limb muscles. These electrophysiological abnormalities that are indicative of active and chronic denervation and of reinnervation and that, according to the Awaji-shima consensus recommendations [1], are equivalent, and therefore additive, to clinical findings, were consistent with the presence of neurogenic changes in two body regions. Somatosensory-evoked potentials were normal, whereas motor- evoked potentials showed a prolonged central motor conduction time for the upper and lower limb muscles, suggesting pyramidal tract involvement. A diagnosis of ALS was made and treatment with riluzole was prescribed. In February 2011, the patient was admitted to a tertiary referral clinic for motor neuron diseases where the diagnosis was confirmed. In the following months, there was no evident progression of weakness and, in November 2011, a new EMG only showed chronic lower motor neuron signs in the upper and lower limb muscles but no active denervation. The symptoms slowly improved and, in January 2012, the patient was admitted to another tertiary referral clinic. Because of the clinical and electrophysiological improvement, ALS was not confirmed, and a diagnosis of cervical myelopathy was suggested even though there was evidence of neither clear-cord compression nor cervical cord lesion on MRI.

In January 2013, the patient came to our attention. A neurological examination revealed slight weakness of the right pectoralis major, extensor indicis proprius, extensor digitorum communis [ $\mathrm{F}=4 / 5$ on the Medical Research Council (MRC) scale], extensor hallucis longus, and extensor digitorum brevis muscles ( $F=3 / 5$ on the MRC scale). Brisk tendon reflexes, ankle clonus, and Babinski and Hoffmann sign were present on the right side. There was no sensory or urinary and bowel dysfunction. Spine MRI confirmed cervical spondylosis at the C3-C4 level, with no evidence of myelopathy. Motor-evoked potentials showed a prolonged central conduction time in both the upper and lower limbs, but more pronounced on the right side. Somatosensory evoked potentials after stimulation of the median and tibial nerves were normal bilaterally.

Electroneuronography revealed a delayed $\mathrm{F}$ wave recorded in the abductor digiti minimi muscle for the stimulation of the right ulnar nerve, while EMG showed chronic denervation in the upper and lower limb muscles. Laboratory tests, including autoimmune screening and a HIV test, were negative. Serum and cerebrospinal fluid (CSF) did not contain Borrelia burgdorferi antibodies. CSF analysis showed 2 leukocytes $/ \mathrm{mm}^{3}, 0$ red blood cells, and a pro- 
Piombo et al.: Cronobacter sakazakii DNA Detection in Cerebrospinal Fluid of a Patient with Amyotrophic Lateral Sclerosis Mimic Syndrome

tein level of $65 \mathrm{mg} / \mathrm{dl}$ (normal range: 15-45). Routine cultures for bacteria and fungi were negative. We also performed a molecular test for the detection of CSF pathogens using polymerase chain reaction (PCR) technology targeting conserved regions of the $16 \mathrm{~S}$ ribosomal (r) RNA sequence and sequencing of the PCR product. One milliliter of CSF was centrifuged at 13,000 rpm for 10 min to pellet bacterial cells. Bacterial DNA was extracted by the EZ1 DNA tissue kit (Qiagen, Düsseldorf, Germany) according to the manufacturer's instructions, starting from $200 \mu \mathrm{l}$ of bacterial pellet suspension. The $16 \mathrm{~S} r R N A$ gene was amplified by PCR, starting from $5 \mu \mathrm{l}$ DNA in $45 \mu \mathrm{l}$ of a PCR mixture containing $1 \times$ PCR gold buffer with $\mathrm{MgCl}_{2}$ (2.0 mM final concentration; Applied Biosystems, Foster City, Calif., USA), $10 \mathrm{mM}$ deoxynucleotide triphosphate (Applied Biosystems), $1.0 \mu \mathrm{M}$ of each primer (27F 5'GAGTTTGATYMTGGCTCAG3' and 556R 5'CTTACGCCCARTRAWTCCG3') [2], and $1.25 \mathrm{U}$ of AmpliTaq Gold DNA polymerase (Applied Biosystems). PCR cycling parameters included an initial denaturation for $10 \mathrm{~min}$ at $94^{\circ} \mathrm{C} ; 35$ cycles of $1 \mathrm{~min}$ at $94^{\circ} \mathrm{C}, 1 \mathrm{~min}$ at $50^{\circ} \mathrm{C}$, and $1 \mathrm{~min}$ at $72^{\circ} \mathrm{C}$; and a final extension for $7 \mathrm{~min}$ at $72^{\circ} \mathrm{C}$. Amplicons were purified with the Qiaquick PCR product purification kit (Qiagen), according to the manufacturer's instructions. The $16 \mathrm{~S}$ rRNA gene was sequenced using both $27 \mathrm{~F}$ and $558 \mathrm{R}$ primers, and the sequencing technology based on fluorescent dye terminator on ABI PRISM 3730 DNA Analyzer sequencer (Applied Biosystems). The nucleotide sequence was compared to sequences present in the GenBank database using the BLAST programs (NCBI/BLAST, http://www.ncbi.nlm.nih.gov). 16S rRNA bacterial PCR assay of CSF identified $C$. sakazakii with a sequence similarity of $99 \%$. Research for CSF neurotropic virus, including CMV, EBV, HSV-1/2, HHV 6/7/ 8, enterovirus, and VZV, was performed by end-point PCR followed by specific microarray hybridization. Molecular identification of neurotropic viruses in CSF tested negative for all pathogens examined.

At the most recent follow-up in April 2014, the patient showed further improvement of his motor deficits and presented only a moderate running impairment.

\section{Discussion}

C. sakazakii infection is a life-threatening cause of meningitis, septicemia, and necrotizing enterocolitis in infants and neonates [2]. At older ages, $C$. sakazakii causes bacteremia, as well as urosepsis and wound infections, particularly in immunocompromised subjects [3]. C. sakazakii has been isolated from a vast range of foods, including cheese, herbs, spices, meat, and especially from powdered milk substitutes.

Can C. sakazakii infection be the cause of ALS-like syndrome in our patient? Rare cases of reversible motor neuron disease have been reported in association with several infective agents, such as B. burgdorferi, Babesia, HIV, and HTLV-1 [4]. The exact mechanism by which these pathogens influence motor neurons is matter of debate. Three possible mechanisms have been suggested: (i) the direct damage of neural cells by the infective neurotrophic microorganism [5], (ii) the neurotoxic effect mediated by bacterial proteins or by the mounted cytokine response [6], and (iii) the autoimmune cross reaction [7]. One of these mechanisms might also have been triggered by $C$. sakazakii infection in our patient. Since the ALS-like syndrome was reversible and no other possible causes were identified, a causal relationship between $C$. sakazakii infection and motor deficits can be hypothesized, even though $C$. sakazakii was demonstrated in CSF only 3 years after symptom onset.

In conclusion, we report that $C$. sakazakii infection in an immunocompetent individual might have triggered a slowly progressive, spontaneously resolving myeloradiculopathy, involving the upper and lower motor neurons at the low cervical level. 
Piombo et al.: Cronobacter sakazakii DNA Detection in Cerebrospinal Fluid of a Patient with Amyotrophic Lateral Sclerosis Mimic Syndrome

\section{Statement of Ethics}

The authors have no ethical conflicts to disclose.

\section{Disclosure Statement}

The authors declare that they have no conflicts of interest to disclose.

\section{References}

1 de Carvalho M, Dengler R, Eisen A, England JD, Kaji R, Kimura J, Mills K, Mitsumoto H, Nodera H, Shefner J, Swash M: Electrodiagnostic criteria for diagnosis of ALS. Clin Neurophysiol 2008;119:497-503.

2 Maiwald M: Molecular Microbiology: Diagnostic Principles and Practice. Washington, ASM Press, 2011.

-3 Lee HA, Hong S, Park H, Kim H, Kim O: Cronobacter sakazakii infection induced fatal clinical sequels including meningitis in neonatal ICR mice. Lab Anim Res 2011;27:59-62.

4 Holy 0, Forsythe S: Cronobacter spp. as emerging causes of healthcare-associated infection. J Hosp Infect 2014;86:169-177.

5 Harvey WT, Martz D: Motor neuron disease recovery associated with IV ceftriaxone and anti-Babesia therapy. Acta Neurolo Scand 2007;115:129-131.

-6 Moulignier A, Moulonguet A, Pialoux G, Rozenbaum W: Reversible ALS-like disorder in HIV infection. Neurology 2001;57:995-1001.

7 Alfahad T, Nath A: Retroviruses and amyotrophic lateral sclerosis. Antiviral Res 2013;99:180-187. 